\title{
Forensic Bite Mark Identification Using Image Processing Methods
}

\author{
Glenn Flora \\ Department of Computer \& \\ Information Science, IUPUI \\ 723 W. Michigan St \\ Indianapolis, IN 46202, USA \\ gflora@gmail.com
}

\author{
Mihran Tuceryan \\ Department of Computer \& \\ Information Science, IUPUI \\ 723 W. Michigan St \\ Indianapolis, IN 46202, USA \\ tuceryan@iupui.edu
}

\author{
Herb Blitzer \\ Indiana Forensic Institute \\ 338 South Arlington Ave, Suite 111 \\ Indianapolis, IN 46219, USA \\ hblitzer@ifi-indy.org
}

\begin{abstract}
Forensic dentistry generally addresses the problem of identifying individuals based on the properties of teeth or identifying individuals based on bite mark impressions. It is legally relevant to accurately and reliably match a bite mark impression to place a criminal at the scene of a crime. Therefore, a system which minimizes human interaction to conduct the comparison would be beneficial to ensure accuracy and reduce human bias. This paper describes experiments with developing a semi-automated method to compare 3D dental models taken from candidate humans and bite mark impression images left in the scene of the crime. Once the contours from the bite mark image and the 3-dimensional dental model are captured, the ideal alignment is calculated by finding the transformation which minimizes a distance measure. The best match is then identified by performing this comparison to a set of candidate dental models. The results are compared to identification results by human forensic odontology experts.
\end{abstract}

\section{Categories and Subject Descriptors}

I.4.6 [Segmentation]: Edge and feature detection; I.4.9 [Image Processing and Computer Vision]: Applications; I.5.4 [Pattern Recognition]: Applications

\section{General Terms}

Algorithms, Experimentation, Legal Aspects

\section{Keywords}

Bite mark identification, 3D teeth models, forensic odontology, Image matching

\section{INTRODUCTION}

Forensic dentistry generally addresses the problem of identifying individuals based on the properties of teeth. By look-

Permission to make digital or hard copies of all or part of this work for personal or classroom use is granted without fee provided that copies are not made or distributed for profit or commercial advantage and that copies bear this notice and the full citation on the first page. To copy otherwise, to republish, to post on servers or to redistribute to lists, requires prior specific permission and/or a fee.

SAC'09 March 8-12, 2009, Honolulu, Hawaii, U.S.A.

Copyright 2009 ACM 978-1-60558-166-8/09/03 ...\$5.00. ing at the location, orientation, presence/absence, and dental work, people can be matched to dental records or bite mark impressions for identification.

One application of forensic dentistry, and the subject of this paper, is identifying individuals based on bite mark impressions, particularly, matching bite marks left on victims to the perpetrator or matching bite marks left on criminals to the victim in instances of violent crime. It is legally relevant to match the bite mark to the person who left it with high confidence. "Bite mark evidence has been almost universally accepted in the courts, but the fundamental validity and scientific basis for its use is frequently challenged [11]."

This paper focuses on the application of partially automated image processing and image matching techniques for identifying criminal suspects using bite marks left in crime scenes. This work may be used as a step towards nearcomplete automation as in the case of fingerprint matching, or the system will work concurrently with forensic odontologists. Two different levels of automation and objectivity is explored with each requiring different user-initializations. In the first method, the user manually identifies the tooth contours in the simulated bite mark image. In the second method, the user approximately identifies the center of each tooth in the simulated bite mark image, and the tooth contour is then extracted by placing a template on the image based on the user input.

\section{RELATED WORK}

There are two main applications of forensic dentistry: (i) identifying deceased individuals by matching ante mortem and post mortem dental records and (ii) identifying criminals based on bite marks $[11,12]$. Typically, identification based on teeth has been done using 2D images such as dental radiographs and the actual teeth or their $2 \mathrm{D}$ images. In [12], Sweet explores methods for identifying criminals based on bite marks. This is done by comparing unique attributes and patterns in the suspect's teeth with similar characteristics in the injury.

In many situations, the identification is done manually on 2D image data by experts using tools such as Adobe Photoshop to obtain the best alignment [13]. In [10], McNamee conducted a comparative reliability study of captured bite mark overlays and showed little variation between the positions of tooth edges captured by different examiners.

Chen and Jain develop a semi-automated approach for the comparison in $[2,8]$. This method uses image registration methods applied to extracted tooth contours in potential 
radiographs to determine if there is a match. More recent work involves using 3D digitized models of teeth, typically obtained using dental casts of the human subjects [3]. Planar cross-sections from 3D digital models are identified manually to be matched against bite mark impressions. Others have used similar approaches to varying extents of automation [7].

Matching of 3D teeth models to 3D bite marks have also been studied [1]. But obtaining 3D bite mark data in real situations is not easy, therefore, the usefulness of such techniques is not clear. Techniques other than $2 \mathrm{D}$ or $3 \mathrm{D}$ data alignment have also been tried [4].

\section{METHOD AND IMPLEMENTATION}

The general steps for bite mark identification are as follows:

1. create a digitization of the set of 3-dimensional dental casts.

2. capture a 2-dimensional contour of the teeth from each digitization.

3. capture the tooth contour from each bite mark image.

4. compare each bite mark image contour to each contour from the dental model by finding the ideal alignment and calculating goodness-of-fit.

5. the comparison which causes the maximum goodnessof-fit is identified as the match.

In the following sections we will describe the details of these steps and the experimental results. Extracting the $2 \mathrm{D}$ contours from a $3 \mathrm{D}$ model is done automatically. We have tried two different methods of obtaining $2 \mathrm{D}$ contours from bite mark images: one manual and one semi-automatic. The matching of the contours from the teeth model to the contours of bite marks is done automatically by finding the best alignment between the two sets.

\subsection{Data collection}

In order to test our method, there were two sets of data collected. One was the collection of 3D teeth models and the second was the collection of bite mark images made by these teeth models.

The Indiana Forensic Institute in Indianapolis (IFI) obtained 15 anonymous dental casts, from the Indiana University School of Dentistry, which were used to test our methods. The casts were 3-dimensional models of volunteers' upper and lower jaws and teeth. Each dental cast was scanned from a distance of $50 \mathrm{~cm}$ to produce a complete 3D digitized model using a Minolta Vivid 910 Non-contact 3D digitizer (see Figure 1 for an example).

The bite mark images were generated using the dental casts. In order to generate bite marks, the tips of the teeth in the $3 \mathrm{D}$ casts were coated with lipstick and the models were pressed upon a soft, foam cushion with $5 \mathrm{lbs}$ of force. The foam cushion was the torso of a child's doll, approximately $7.5 \mathrm{~cm}$ in diameter. Three bite mark simulations per model were performed. The resulting bite marks were then photographed as a $2 \mathrm{D}$ digital color image. Figure 2 shows a bite mark image sample generated by this process.

\subsection{Obtaining 2D Contours from the 3D Den- tal Model}

The 2D teeth contours are obtained from the 3D dental model by intersecting it with a plane. All possible heights

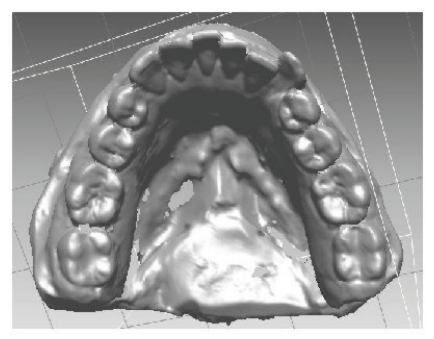

Figure 1: A typical digitized dental model imported into Rapidform editing software.

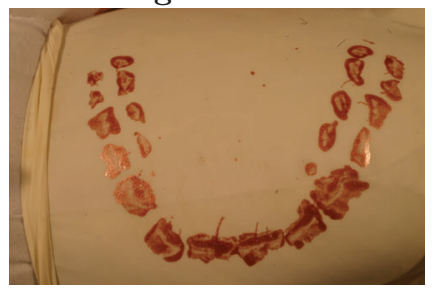

Figure 2: An example of the bite mark image generated by the dental casts.

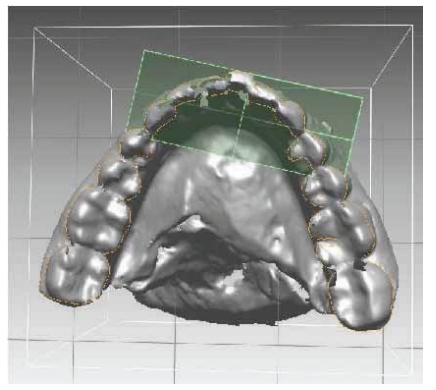

Figure 3: Digitized 3D dental model with intersecting plane and captured tooth contour.

and angles for such planes are tried and the one with the maximum number of line segments in the intersection is selected as the cross-section best representing the teeth, capturing the complex details of the teeth of this person.

The model is oriented lengthwise along the $y$-axis with the teeth in the $x-z$ plane approximately symmetric about the $x$ axis. The initial height of the intersecting plane is set using the bounding box of the model. The plane is varied then by increments of $0.25 \mathrm{~mm}$ and for each height by increments of $\pm 2.5^{\circ}$. An example is shown in Figure 3.

\subsection{Extracting 2D Contours from the Bite Mark Images}

The 2D contours from the bite marks are more difficult to extract due to the quality of the bite mark images. We experimented with two methods to help the forensic analyst with this task. The first approach lets a human expert interactively draw the teeth contours on the image. As expected, this is a time-consuming task and even then, with some bite mark images, it is sometimes difficult to know where exactly the true bite mark contour is supposed to be.

The second approach we used automates this process to a degree. In this approach the human expert indicates each of the teeth by clicking approximately in their centers. The contours the are identified by placing an oval shaped energy 
minimizing snake on these points, and deforming the snake curve according to the image data and an internal potential energy of the snake.

\subsubsection{Manual Bite Mark Contour Extraction}

The first method is a simple, manual method in which the user interactively draws the edge around each tooth in the bite mark image using any image editing software. To capture the edge contours for this project, each bite mark image was opened in Adobe Photoshop. A transparent layer was then created over the original bite mark image, and the tooth edges were traced onto the transparent layer. The layer containing only the tooth edges (teeth contour) was saved as a separate binary image. In extracting the teeth contour, the correct placement of the edge seems to be less important. The most important aspects of the contour retrieval are to capture the relative position of the teeth, the approximate shape and size of each tooth, and the orientation of each tooth.

\subsubsection{Semi-automated Bite Mark Contour Extraction Using Deformable Curves}

The second method for extracting the teeth contours from the bite mark image provides a greater level of automation. This method utilizes a template which is initially placed on the bite mark image by the user interactively. The user approximately identifies the center of each tooth in the bite mark image by clicking on the corresponding location on the image. The locations of the tooth centers do not need to be identified precisely; minimizing the energy of the deformable contour will automatically adjust the contour to the image data. The template is then appropriately scaled to match the bite mark image based on these tooth center positions (Figure 4). The scaling is based on the average of two width and two height measurements and results in individual teeth of appropriate size. The template acts as the initial configuration for an Active Deformable Contour algorithm which manipulates the template to match the tooth contours for that particular bite mark image.

Once the teeth positions and the template is initialized, the contours of teeth are deformed to achieve a minimum energy configuration based on image forces and internal potential energy as described in [9]. The snake energy is calculated based on the continuity, curvature, and the inherent image energy. Representing the position of the snake parametrically by $\mathbf{v}(s)=(x(s), y(s))$, the energy of the snake is calculated as:

$$
E_{\text {snake }}=\int\left(E_{\text {int }}(\mathbf{v}(s))+E_{\text {image }}(\mathbf{v}(s))+E_{\text {con }}(\mathbf{v}(s))\right) d s
$$

$E_{\text {int }}$ represents the internal energy of the snake due to bending and stretching, $E_{\text {image }}$ represents forces due to the image and is based on the high gradient points in the bite mark image that pull the contour toward them, and $E_{\text {con }}$ represents the energy due to control points. Since we do not have interactive control points to move the contour, we have omitted this term in our implementation. The remaining two energy terms as used in our work are given as follows:

$$
\begin{aligned}
E_{\text {image }} & =-\gamma|\nabla I(x, y)|^{2} \\
E_{\text {int }} & =\frac{1}{2}\left(\alpha\left|v_{s}(s)\right|^{2}+\beta\left|v_{s s}(s)\right|^{2}\right)
\end{aligned}
$$

The values of the coefficients in our work are set (empirically) as $\alpha=1, \beta=15$, and $\gamma=5$. Figure 5 gives some
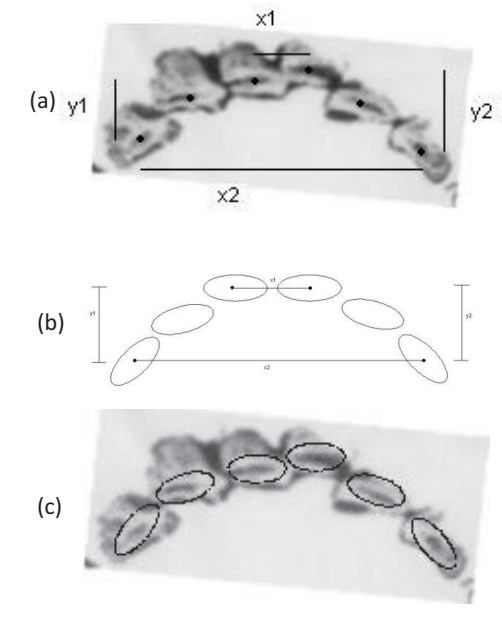

Figure 4: Scaling of the template for placement on the bite mark image (a) user identification of tooth centers and measurements used for scaling (b) corresponsing measurements on the template, and (c) the initial template placement.
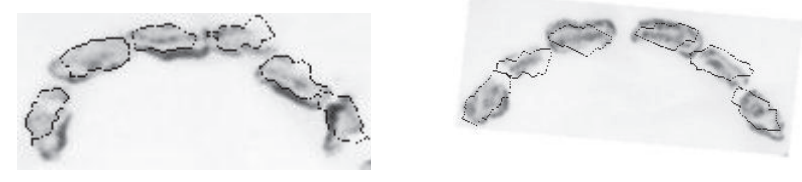

Figure 5: Typical captured tooth contours using the deformable curve.

typical results of the bite mark contours found.

In order to apply the matching algorithms, we fill the detected bite mark image contours. This is done by the recursive 4-connected, flood-fill algorithm described in [6].

\subsection{Contour Matching Algorithm and Com- puting the Goodness-of-Fit}

Once the 2D contours from the 3D model and the bite mark images are obtained as described in the previous section, we then find the best match between the 2D bite mark image contour and each of the candidate model contours. The matching between the contours is computed by finding the best $2 \mathrm{D}$ alignment according to the goodness-of-fit measure defined in this section. This search for the best alignment is done over the rigid $2 \mathrm{D}$ transformation space. The goodness-of-fit measure consists of two parts that are combined to obtain an overall measure. One part is based on a chamfer distance measure and the second part is based on a measure of the amount of overlap of the two filled contours.

The chamfer distance was calculated as given by Gavrila in [5] and was used for the contour matching. Given two point sets $U=\left\{u_{i}\right\}, i=1, \ldots, n$ and $V=\left\{v_{j}\right\}, j=1, \ldots, m$, the chamfer distance function is the distance between each point, $u_{i} \in U$ and its closest point in $\mathrm{V}$ :

$$
d_{\text {chamf }}(U, V)=(1 / n) \sum_{u_{i} \in U}\left(\min _{v_{j} \in V}\left\|u_{i}-v_{j}\right\|\right)
$$


where $\|\cdot\|$ is the Euclidian distance and $n$ is the number of points in $U$. For the ideal alignment between the two contours, $U$ corresponds to the bite mark contour and $V$ corresponds to the contour from the dental model. The chamfer distance is a per pixel error measurement, and the ideal alignment will minimize the error.

The second part of the goodness-of-fit measure is based on the amount of overlap between the two contours after they are aligned for the best match. This measurement was computed as the fraction of overlap between the filled bite mark contour and the filled dental model contour. The overlap measurement is computed as:

$$
d_{\text {overlap }}\left(U_{\text {fill }}, V_{\text {fill }}\right)=\frac{\left|U_{\text {fill }} \bigcap V_{\text {fill }}\right|}{\left|U_{\text {fill }}\right|}
$$

where, $|S|$ is the cardinality of $S$ and in our case represents the number of pixels in the set. The computed overlap measure will be 1 for a perfect alignment and approach 0 for a poor alignment.

Once the translation was found for the ideal alignment, the chamfer distance was computed and normalized. The $d_{\text {chamf }}$ was approximately 3 for a typically good alignment, but was greater than 10 for a poor alignment. Thus, a normalized overall goodness measure based on $d_{\text {chamf }}$ was computed as

$$
\text { goodness }_{\text {chamf }}=\left\{\begin{array}{cl}
1-d_{\text {chamf }} / 10 & \text { if } d_{\text {chamf }}<10 \\
0 & \text { if } d_{\text {chamf }}>10
\end{array}\right.
$$

The overall goodness-of-fit was calculated as a linear combination of the two distances:

$$
\text { goodness-of-fit } \alpha \times d_{\text {overlap }}+\beta \times \text { goodness }_{\text {chamf }}
$$

The values of $\alpha$ and $\beta$ were determined empirically through experimentation. It was determined that finding the initial alignment maximizing the overlap amount was most successful $(\alpha=1, \beta=0)$. Once the best alignment of the bite mark contour with the dental model contour is found, the result was refined and the overall goodness-of-fit was computed by including the $d_{\text {chamf }}$ with $\alpha=0.25$ and $\beta=0.75$.

\section{EXPERIMENTAL RESULTS AND DISCUS- SION}

Our method of dental identification was tested on a set of $153 \mathrm{D}$ dental models with 2 or 3 bite mark images for each model for a total of 42 bite mark images. We asked two forensic odontologists to visually identify and match the bite mark images against the physical dental casts. The data presented to the forensic odontologists consisted of the dental casts that were scanned to generate the 3D dental models and a set of multiple bite mark images from each dental model. Each group of bite marks had to be matched to the same model, so there were two or three repetitions to be used together to make the match. The forensic odontologists used the same scaled bite mark images used in our automated matching tests. The identity of the bite marks were known, but the identity of the dental casts were unknown. They did not make impressions with the 3-dimensional models but visually compared the 3 -dimensional shape to the 2-dimensional bite mark images. The performance of the two human experts was used as a benchmark for the identification results of our two methods. The two forensic odontologists correctly matched the group of bite marks to the corresponding dental model $73 \%$ and $60 \%$ of the time, respectively. The overall rate of correct matches was $67 \%$. The bite mark was always identified correctly $47 \%$ of the time, correctly identified at least once $87 \%$ of the time, and never identified correctly $13 \%$ of the time.

\subsection{Automated matching results}

The algorithms described in Sections 3.2-3.4 were tested on the set of 15 3D teeth models and 42 bite mark images. In this experiment, we only used the front 6 teeth in order to identify the bite marks. This was justified after consulting with the forensic odontologists that most bite marks in crime scenes would involve the front teeth. In our tests, each bite mark was treated as being independent from any other bite mark, including those within the same group generated by the same 3D dental cast. This differs from the forensic odontologists' comparison because the automated matching does not have the advantage of utilizing combined information from the repeated bite marks. Therefore, for the automated method, there are 42 separate bite marks to be matched to the 15 tooth models.

We ran the identification algorithm using the manual extraction of bite mark contours and automated matching (method 1) and using the deformable model based extraction of bite mark contours and automated matching (method 2).

Using method 1, the matching and goodness-of-fit results on all 15 models and 42 bite mark images is summarized in Figure 6. In this figure, each column represents the goodness-of-fit measure of this particular bite mark image against the 15 possible 3D models. The larger circles represent what should have been identified as the correct match. The smaller squares represent what should have been identified as an incorrect match. Ideally, all the larger circles should be at the top of each column, which would correspond to $100 \%$ correct identification.

The automated method missed a total of five comparisons over four different bite mark groups. Of the four missed groups by the automated method, two were always identified correctly by the forensic odontologists and two were correctly identified once. All of the correct matches received a high goodness-of-fit measurement, but the algorithm found a slightly better match that was incorrect.

In these tests, overall, method 1 was an improvement over the forensic odontologists' comparison. This method correctly matched $88 \%$ of the time while the forensic odontologists matched correctly $67 \%$ of the time. Also, the method 1 always identified the bite mark correctly $73 \%$ of the time while the forensic odontologists always matched correctly $47 \%$ of the time.

Using method 2, the matching and goodness-of-fit results on all 15 models and 42 bite mark images is summarized in Figure 7. As in method 1 tests, each bite mark is treated independently, so there are 42 different bite marks to participate in the comparison. The larger circles represent correct matches, and the smaller squares represent incorrect matches. Overall, the algorithm correctly matched a bite mark to the corresponding dental model $52 \%$ of the time when the template was placed by the user.

There was no notable correlation between the correct and incorrect matches between this method and the forensic odontologists' comparison. Assuming the correct size of each tooth in the bite mark contour, the success of the iden- 
Bite Mark - Dental Model Goodness-of-Fit

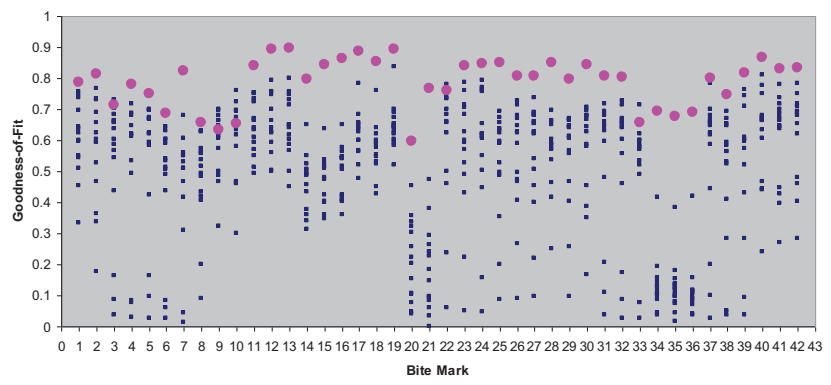

Figure 6: Matching results for the comparison using manually extracted contours from the bite mark images.

Bite Mark - Dental Model Goodness-of-Fit

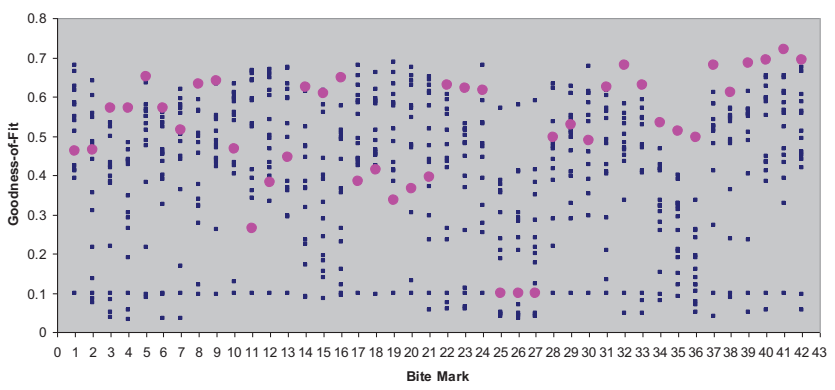

Figure 7: Matching results for the comparison using bite mark contours extracted with deformable curves.

tification is influenced by the general shape, position and orientation.

\section{CONCLUSIONS}

In this paper, we presented a computational framework for forensic identification based on bite marks. Two semiautomated methods were developed and tested for comparing bite marks to 3 -dimensional candidate dental models. The results of the two methods were then compared to a benchmark set by the same comparison conducted by a two forensic odontologists.

Using the manually extracted the contours from bite mark images and using automated matching methods improved the identification results compared to forensic odontologists' results. Using the automated contour extraction from bite mark images and automated matching did not perform as well as the first method. This was partly due to the faults in the extracted contours from the bite mark images and this needs to be improved. Part of the reason for poor performance of the contour extraction in method 2 was due to the way the bite mark images were generated from the dental casts. If a more realistic method of bite mark generation is used, the automated method results would also improve. We did not use any real bite mark data from forensic cases. The use of such data would also be an interesting experiment for future testing.

\section{ACKNOWLEDGMENTS}

This work was supported by the US National Institute of Justice grant \#2006-DN-BX-K252. We gratefully acknowledge Dr. Edwin T. Parks and Dr. Jeffrey A. Platt from the Indiana University School of Dentistry for providing the dental casts and helping us with the human expert bite mark identifications.

\section{REFERENCES}

[1] S. A. Blackwell, R. V. Taylor, I. Gordon, C. L. Ogleby, T. Tanijiri, M. Yoshino, M. R. Donald, and J. G. Clement. 3-D imaging and quantitative comparison of human dentitions and simulated bite marks. International Journal of Legal Medicine, 121(1):9-17, 2007. 10.1007/s00414-005-0058-6.

[2] H. Chen and A. K. Jain. Dental biometrics: alignment and matching of dental radiographs. IEEE Transactions on Pattern Analysis and Machine Intelligence, 27(8):1319-1326, 2005.

[3] T. Dharmadhikari. Forensic odontology using digital geometry analysis. MS Thesis, Purdue University, 2006.

[4] G. Fahmy, D. Nassar, E. Haj-Said, H. Chen, O. Nomir, J. Zhou, R. Howell, H. H. Ammar, M. Abdel-Mottaleb, and A. K. Jain. Towards an Automated Dental Identification System (ADIS). In Proceedings of the International Conference on Biometric Authentication (ICBA), Hong Kong, 2004.

[5] D. M. Gavrila. A bayesian, exemplar-based approach to hierarchical shape matching. IEEE Transactions on Pattern Analysis and Machine Intelligence, 29(8):1408-1421, 2007.

[6] D. Hearn and M. P. Baker. Computer Graphics. Prentice Hall, New Jersey, 2004.

[7] S. M. d. 1. Heras, A. Valenzuela, C. Ogayar, A. J. Valverde, and J. C. Torres. Computer-based production of comparison overlays from 3D-scanned dental casts for bite mark analysis. Journal of Forensic Sciences, 50(1):127-133, 2005.

[8] A. K. Jain and H. Chen. Matching of dental X-ray images for human identification. Pattern Recognition, 37(7):1519-1532, 2004.

[9] M. Kass, A. Witkin, and D. Terzopoulos. Snakes: Active contour models. International Journal of Computer Vision, 1(4):321-331, Jan. 1988.

[10] A. H. McNamee, D. Sweet, and I. Pretty. A comparative reliability analysis of computer-generated bitemark overlays. Journal of Forensic Science, 50(2):400-405, 2005.

[11] I. A. Pretty and D. Sweet. Digital bite mark overlays - an analysis of effectiveness. Journal of Forensic Science, 46(6):1385-1390, 2001.

[12] I. A. Pretty and D. Sweet. A look at forensic dentistry - part 1: The role of teeth in the determination of human identity. British Dental Journal, 190(7):359-366, 2001.

[13] D. Sweet. Computer assisted 2-d and 3-d comparison of bite mark evidence and tooth exemplars. Technical Report TR-07-98, Bureau of Legal Dentistry, University of British Columbia, 1997. 